\title{
Análisis de la calidad de los efluentes de los biodigestores en los lodges ubicados en la zona alta de la Reserva de Producción de Fauna Cuyabeno
}

\section{(Quality analysis of effluents from biodigesters in lodges located in the Cuyabeno Wildlife Reserve)}

\author{
Isidro Gutiérrez Cruz ${ }^{1}$, Edgar Rivera Carrión ${ }^{1}$, Gloria Roldán Reascos ${ }^{1}$
}

\begin{abstract}
Resumen:
En la Reserva de Producción de Fauna Cuyabeno, sectores Laguna Grande y río Cuyabeno, se han construido hoteles ecológicos, para atender la demanda turística, los mismos que generan aguas residuales. El Ministerio de Ambiente, responsable de la conservación de la biodiversidad, ha dispuesto la implementación de biodigestores, para el tratamiento de estas aguas. Después de tres años de implementación del sistema, se propone evaluar la calidad de estas aguas, a través del análisis de parámetros físico-químicos: temperatura, conductividad, $\mathrm{pH}$, sulfatos, nitratos y nitritos, detergentes, $\mathrm{DQO}, \mathrm{DBO}_{5}$, aceites y grasas; y microbiológicos: coliformes fecales; cuyos resultados fueron comparados con la legislación ambiental vigente: TULSMA "Límites de descarga a un cuerpo de agua dulce". La toma de muestras se sujetó a lo que establece la Norma INEN 2 169:2013 y se muestreó en la época de alta y de baja pluviosidad. Los resultados determinaron que los biodigestores únicamente cumplen la norma en tres parámetros: nitratos y nitritos; sulfatos y temperatura; e incumplen la norma en: $D B O_{5}$, $D Q O$ y $\mathrm{pH}$; mientras que en los cuatro parámetros restantes, existe una variación de los resultados. En conclusión todos los biodigestores, incumplen con la normativa, al menos en uno de los parámetros.
\end{abstract}

Palabras clave: Biodigestores; Aguas residuales domésticas; Efluentes.

\begin{abstract}
:
In the Cuyabeno Wildlife Reserve near Laguna Grande and Cuyabeno river, several ecological hotels have been built to meet the touristic demand; therefore these eco-lodges generate wastewaters. The Ministry of Environment, responsible for biodiversity conservation, has ordered the implementation of biodigesters for the treatment of these waters. After three years of implementing the system, it is proposed to check the quality of these waters, through the analysis of physical-chemical parameters: temperature, conductivity, $\mathrm{pH}$, sulfates, nitrates and nitrites, detergents, COD, BOD5, oils and fats; and microbiological such as faecal coliforms. These results were compared with the current environmental legislation: TULSMA "Limits discharged to a body of fresh water." Sampling was subject to the provisions of standard Regulation INEN 2 169: 2013 and sampled at the time of high and low rainfall. The results determined that biodigesters only meet the standard regulations in three parameters: nitrates and nitrites; sulfates and temperature; and violate the following regulations: BOD5, COD and $\mathrm{pH}$; while in the remaining four parameters, there is a variation among the results. In conclusion all digesters violate the regulations, at least on one of the parameters.
\end{abstract}

Keywords: Biodigesters; domestic wastewater; effluents.

\footnotetext{
${ }^{1}$ Universidad Tecnológica Equinoccial, Quito - Ecuador ( \{isidro.gutierrez, franco.rivera, gloria.roldan\} @ute.edu.ec )
} 


\section{Introducción}

La Reserva de Producción de Fauna Cuyabeno (RPFC), se crea mediante Acuerdo Ministerial Nro. 0322 en julio de 1979, con una superficie de 254.760 ha. Posteriormente se amplían y modifican sus límites, mediante varios Acuerdos Ministeriales en 1991 y 1994. (Ministerio del Ambiente, 2012). Finalmente, mediante Acuerdo Ministerial Nro. 188, publicado en el Registro Oficial Nro. 880 de 28 de enero de 2013, se definen los nuevos límites de la Reserva, con lo cual la superficie aproximada actual es de 590.112 hectáreas.

Se encuentra localizada al noroeste del territorio ecuatoriano, en las provincias de Sucumbíos y Orellana. En su parte occidental, la Reserva presenta una zona accidentada con colinas bajas, y rápidamente el terreno se vuelve más plano hacia el oriente donde existen dos llanuras muy planas inundables, que dan lugar a la formación de sistemas de lagunas interconectadas (Robbins \& Long, 2010).

Contiene el mayor sistema lacustre tropical del Ecuador, está atravesada de oeste a este por los ríos Cuyabeno y Aguarico, que forman un sistema de 14 lagunas comunicadas entre sí por pequeños canales, de las cuales las más sobresalientes son: Laguna Grande, Caimancocha, Patacocha y la Quebrada de la Hormiga. Otro sector es el complejo del río Lagarto formado por los ríos Imuya y Lagarto, las lagunas: Lagartococha, Imuya, Delfincocha y otras de menor tamaño. El agua del Aguarico es de las que se denomina "Blanca" y contiene alta cantidad de sedimentos que trae desde los Andes. (Andrade, 2010).

La Reserva es uno de los principales destinos turísticos de la Amazonía ecuatoriana, con elevada afluencia de visitantes, por ser un Área Protegida que mantiene una gran diversidad de ecosistemas y especies de flora y fauna, que la ubican como una de las zonas más biodiversas del planeta. De igual manera el Área Protegida posee un gran valor cultural ya que incluye las nacionalidades indígenas ancestrales: Siona, Secoya, Cofán, Kichwa y Shuar (ECOLAP-MAE, 2007). La actividad turística se desarrolla principalmente en los alrededores del río Cuyabeno y la Laguna Grande que es uno de los sitios de visita preferidos por los turistas.

El Ministerio de Ambiente, organismo rector de la gestión ambiental y de la conservación y manejo de las Áreas Protegidas, ha desarrollado un proyecto para reducir la contaminación provocada por la operación turística, que consiste en la implementación de biodigestores en cada uno de los lodges, para tratar las aguas residuales antes de que estas sean descargadas a la laguna y río Cuyabeno. Tres años después de implementado este sistema, se propone realizar un monitoreo, con el apoyo de la UTE, para conocer la calidad del agua que está siendo descargada a los sistemas hídricos de la Reserva. 
La investigación se realizó en nueve lodges ubicados en la zona alta de la Resera de Producción de Fauna Cuyabeno, donde se han instalado biodigestores para el tratamiento de sus aguas negras y grises. La gestión operativa de los nueve lodges, en campo, se encuentra a cargo de 39 personas, responsables de la atención a turistas y mantenimiento de las instalaciones.

Las aguas servidas generadas por la actividad turística en la parte alta del río Cuyabeno y alrededor de la Laguna Grande, son tratadas a través de biodigestores instalados en los diferentes lodges, los cuales requieren un monitoreo permanente, para garantizar su efectivo funcionamiento, antes de que los efluentes sean vertidos al ecosistema.

Los objetivos de esta investigación fueron evaluar las características de las descargas de aguas residuales producidas por la actividad turística, las mismas que son procesadas a través de los biodigestores instalados en cada lodge y proponer correctivos a los sistemas de biodigestores, para mejorar su funcionamiento.

\section{Metodología}

\section{Materiales y equipos utilizados}

Los materiales a utilizarse fueron preparados tomando en cuenta la norma INEN 2 169:2013, tanto para las muestras in situ, como para la preservación de las mismas, a fin de asegurar la veracidad de los resultados.

Con este propósito, se utilizaron los siguientes materiales y equipos:

- Botellas de $2 \mathrm{~L}$ plásticas previamente esterilizadas para análisis de $\mathrm{DBO}_{5}, \mathrm{DQO}$, nitratos, nitritos, detergentes y sulfatos.

- Botellas de vidrio boca grande de $1 \mathrm{~L}$ previamente esterilizadas para análisis de aceites y grasas.

- Envases de $100 \mathrm{~mL}$ plásticos previamente esterilizados para análisis de coliformes fecales.

- Coolers con capacidad de 20L para transporte de las muestras, con sus respectivos geles refrigerantes para mantener una temperatura adecuada.

- Guantes de caucho

- Agua destilada

- pH-metros portátiles Hanna pHep

- Conductímetros portátiles Hanna DiST 4

- Termómetro digital 
- GPS

- Equipo fotográfico

\section{Etiquetado del material}

Los materiales fueron previamente etiquetados para la identificación de cada muestra. Para cada lodge se utilizó la numeración del 1 al 9, FQ y MB (físico químicos y microbiológicos, respectivamente) y la diferenciación ".1" o ".2" según el biodigestor.

\section{Plan de Muestreo}

El Plan de muestreo incluyó: la frecuencia de muestreo, los puntos de monitoreo, la medición de los parámetros, y la generación de fichas informativas de cada punto monitoreado. Se realizaron dos muestreos, uno en la época de alta pluviosidad, del 12 al 14 de octubre de 2014; y el segundo en época de baja pluviosidad, del 6 al 8 de febrero de 2015.

La toma de muestras, almacenamiento y transporte, de las mismas, en los dos muestreos, se realizó según lo establecido por la Norma INEN 2 169:2013, sobre Calidad del agua, muestreo, manejo y conservación de muestras (INEN, 2013).

En el primer muestreo se tomó el criterio de escoger dos biodigestores por lodge:

B1 "biodigestores 1", donde la mezcla de las aguas de descarga con las aguas del entorno tiene una probabilidad baja.

B2 "biodigestores 2", donde la mezcla de aguas de descarga de los biodigestores, con las de la Laguna Grande o el Río, tiene una probabilidad alta.

Las muestras de agua fueron tomadas a la salida del biodigestor, en las cajas de revisión. Adicional a esto, se realizó la medición de parámetros in situ, los cuales fueron: $\mathrm{pH}$, Conductividad y Temperatura.

Para el segundo muestreo se tomó el criterio de escoger los biodigestores que obtuvieron los peores resultados en el primer muestreo, para evaluar su eficiencia; por tanto, las muestras de agua fueron tomadas antes del ingreso con el biodigestor y a la salida del mismo. También se realizó la medición de parámetros in situ: pH, Conductividad y Temperatura. 


\section{Análisis de muestras}

Para determinar la calidad del agua de descarga de los biodigestores, se analizaron diez parámetros: siete en laboratorio: aceites y grasas, $\mathrm{DBO}_{5}, \mathrm{DQO}$, nitratos y nitritos, detergentes, sulfatos y coliformes fecales; y, tres parámetros in situ: $\mathrm{pH}$, Temperatura y Conductividad.

Todos ellos fueron comparados con la legislación aplicable: Texto Unificado de Legislación Secundaria de Medio Ambiente, Libro VI; Anexo 1: Norma de Calidad Ambiental y de Descarga de Efluentes: Recurso Agua; "Límites de descarga a un cuerpo de agua dulce", emitidos según Acuerdo Ministerial Nro. 097-A, (MAE, 2015); a excepción de Conductividad, que no consta en esta normativa, por lo cual se comparó con una escala de aguas naturales de la norma internacional (EPA, 1999).

Los análisis se realizaron en el Laboratorio de Química de la Facultad de Ciencias Químicas de la Universidad Central del Ecuador. Este laboratorio está acreditado bajo la Norma NT INEN ISO/IEC 17025. Las primeras nuestras se analizaron del 15 al 29 de octubre de 2014; y las segundas, del 9 al 26 de febrero de 2015.

\section{Información complementaria}

Se realizó la localización geográfica de cada sitio muestreado mediante GPS; además, se generaron fichas informativas de cada lodge, mediante entrevistas personales a los administradores o encargados de los mismos.

\section{Resultados y Discusión}

\section{Ubicación de los lodges y cantidad de biodigestores}

La infraestructura turística consta de nueve lodges ubicados en las márgenes del Río Cuyabeno y alrededor de la Laguna Grande (Figura 1), los cuales cuentan con sistemas de biodigestores para el tratamiento de las aguas servidas. En la Tabla 1 se detallan las coordenadas de ubicación y los biodigestores con que cuenta cada uno de ellos 


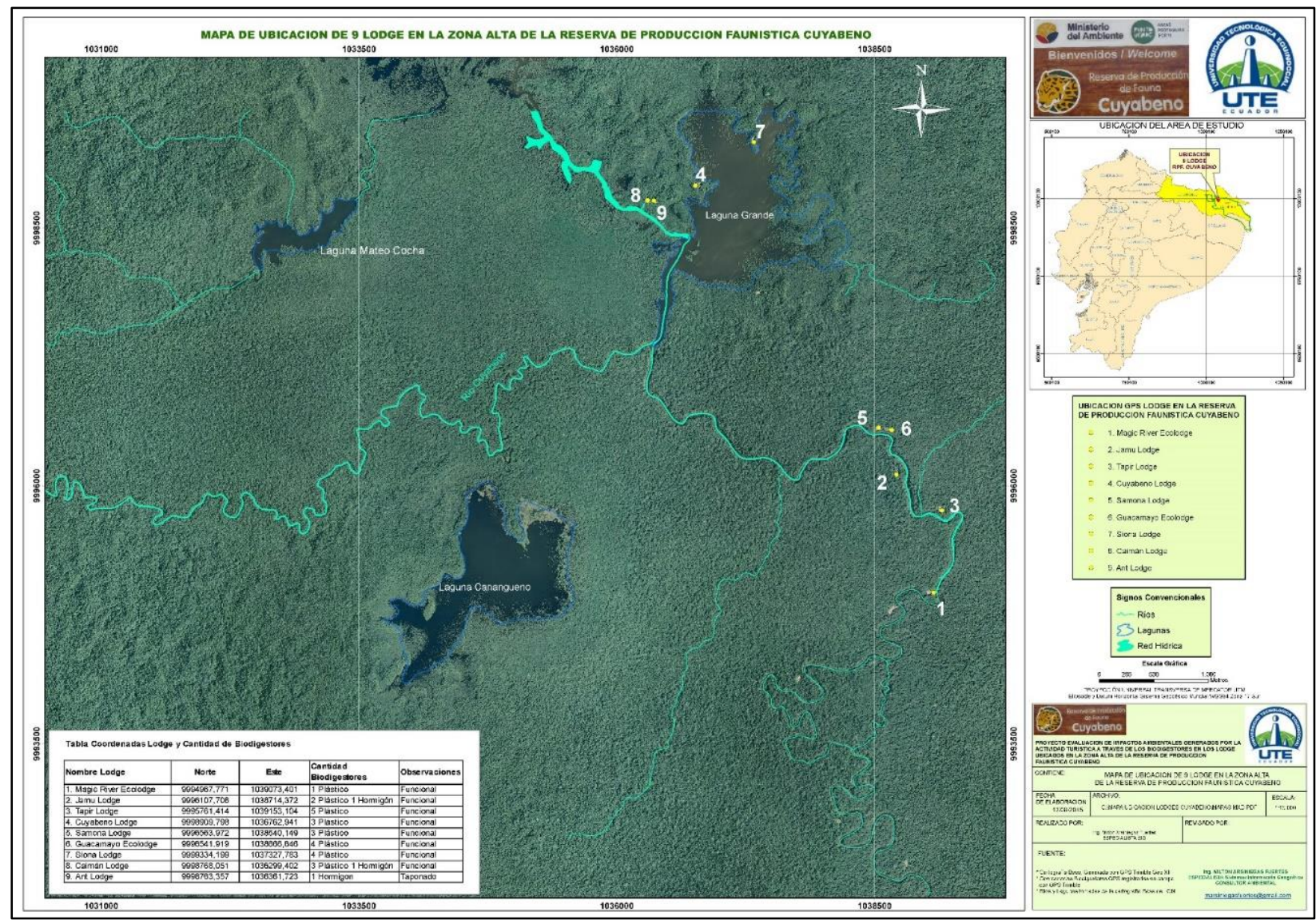

Figura 1. Mapa de ubicación de los Lodges

Fuente: Cartografía Base del IGM, Coordenadas registradas en campo. Elaboración: M. Arsiniegas

Tabla 1. Ubicación geográfica y número de biodigestores de cada Lodge

\begin{tabular}{lcclll}
\hline \multirow{2}{*}{ Nombre Lodge } & \multicolumn{2}{c}{ Coordenadas UTM } & & Cantidad de & Observa- \\
& (Datum WGS84 Zona 17 Sur & & Bodigestores & ciones \\
\cline { 2 - 3 } & Norte & Este & & \\
\hline 1. Magic River Ecolodge & 9994967,771 & 1039073,401 & 2 Plástico & Funcional \\
\hline 2. Jamu Lodge & 9996107,708 & 1038714,372 & 2 Plástico, 1 Hormigón & Funcional \\
\hline 3. Tapir Lodge & 9995761,414 & 1039153,104 & 5 Plástico & Funcional \\
\hline 4. Cuyabeno Lodge & 9998909,798 & 1036762,941 & 3 Plástico & Funcional \\
\hline 5. Samona Lodge & 9996563,972 & 1038540,149 & 4 Plástico & Funcional \\
\hline 6. Guacamayo Ecolodge & 9996541,919 & 1038666,646 & 4 Plástico & Funcional \\
\hline 7. Siona Lodge & 9999334,199 & 1037327,783 & 4 Plástico & Funcional \\
\hline 8. Caimán Lodge & 9998768,051 & 1036299,402 & 5 Plástico, 1 Hormigón & Funcional \\
\hline 9. Hormiga Lodge & 9998763,357 & 1036361,723 & 1 Tri-cameral de hormigón & Taponado \\
\hline
\end{tabular}




\section{Resultados de los análisis de aguas en los biodigestores examinados}

\section{Primer muestreo, Biodigestores B1}

Cuyabeno Lodge es el único que cumple con la normativa en todos los parámetros, en el biodigestor B1, así como nitratos y nitritos, sulfatos y temperatura, que se cumplen en todos los lodges. En los otros biodigestores B1 el cumplimiento es variable, como se observa en la Tabla 2.

Tabla 2. Resultados del primer muestreo, biodigestores 1

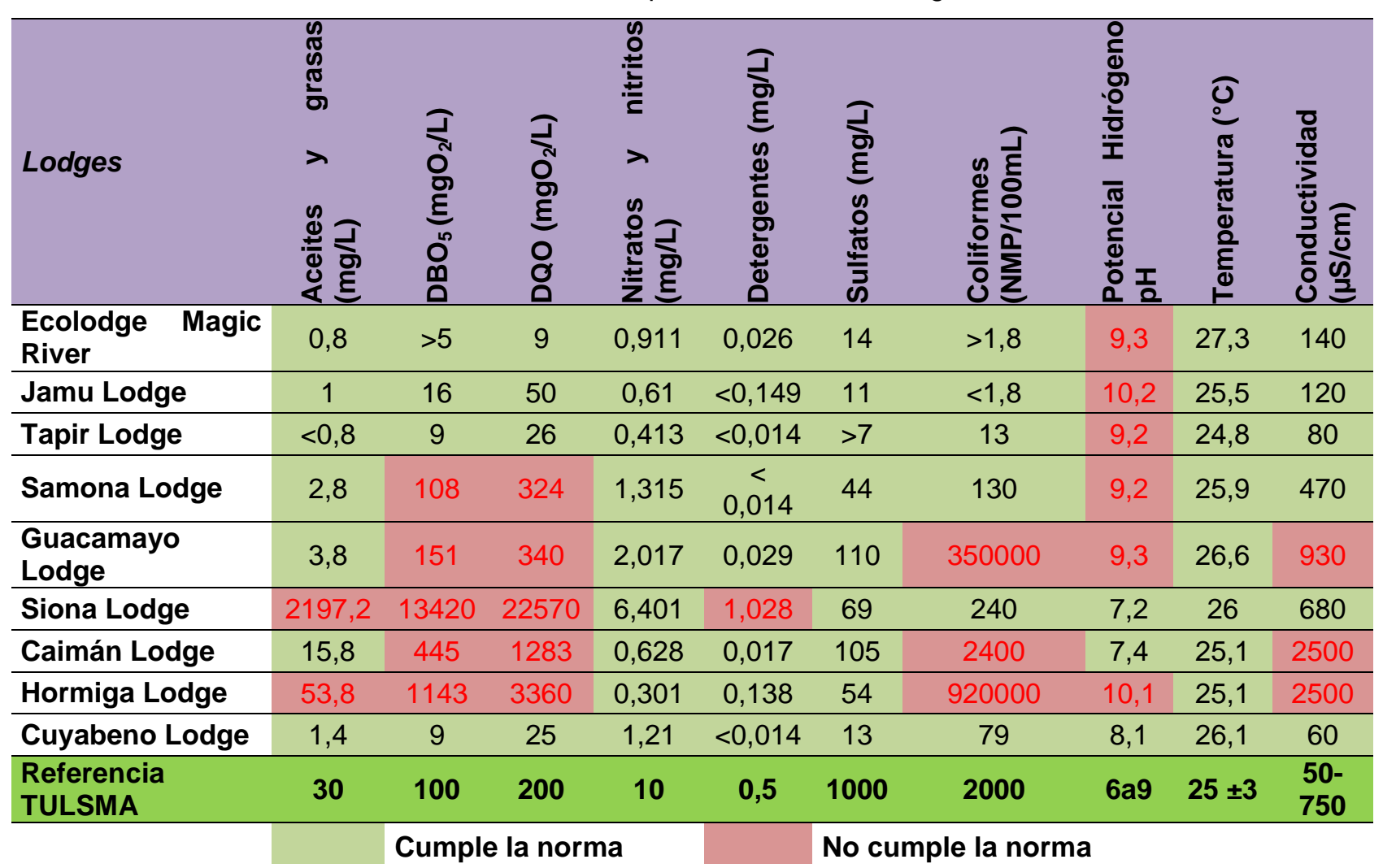

\section{Primer muestreo, Biodigestores B2}

Tapir Lodge es el único que cumple con la normativa en todos los parámetros, en el biodigestor B2, así como aceites y grasas, nitratos y nitritos, detergentes, sulfatos y temperatura, que se cumplen en todos los lodges. En los otros biodigestores B2 el cumplimiento es variable, como se presenta en la Tabla 3.

En el caso de Hormiga Lodge no se incluyen datos de B2, en vista que existe un solo biodigestor. 
Tabla 3. Resultados del primer muestreo, biodigestores 2

\begin{tabular}{|c|c|c|c|c|c|c|c|c|c|c|}
\hline Lodges & 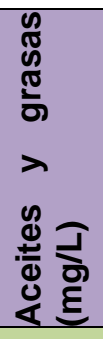 & 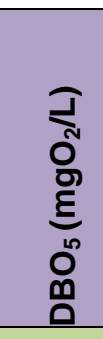 & $\begin{array}{l}\text { J } \\
\text { O } \\
\text { o } \\
\text { है } \\
0 \\
0 \\
0\end{array}$ & 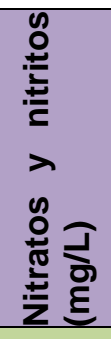 & 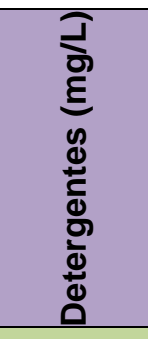 & $\begin{array}{l}\overline{1} \\
\text { है } \\
\text { है } \\
0 \\
\frac{0}{\pi} \\
\frac{\pi}{5} \\
0 \\
0\end{array}$ & 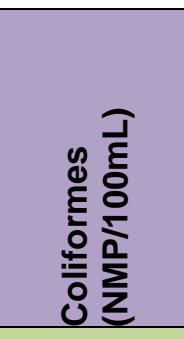 & 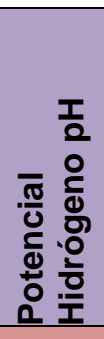 & 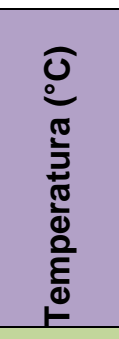 & 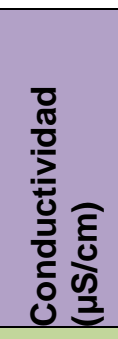 \\
\hline $\begin{array}{l}\text { Ecolodge Magic } \\
\text { River }\end{array}$ & $>0,8$ & 12 & 29 & $>0,2$ & $<0,014$ & 15 & $>1,8$ & 9,5 & 27,9 & 140 \\
\hline Jamu Lodge & $<0,8$ & $<5$ & 14 & 2,39 & $<0,014$ & 26 & 33 & 9,9 & 24,2 & 190 \\
\hline Tapir Lodge & 0,8 & 16 & 48 & 3,803 & $<0,014$ & $<7$ & 170 & 8,9 & 24,9 & 90 \\
\hline Samona Lodge & 7,2 & 227 & 682 & 2,915 & $<0,014$ & 59 & 920 & 8,7 & 26,9 & 540 \\
\hline $\begin{array}{l}\text { Guacamayo } \\
\text { Lodge }\end{array}$ & 0 & - & - & - & - & - & 35000000 & 8,8 & 27,4 & 1870 \\
\hline Siona Lodge & 6 & 142 & 356 & 2,413 & $<0,014$ & 48 & 170000 & 2,3 & 26,3 & 1260 \\
\hline Caimán Lodge & 0,8 & 18 & 53 & 0,717 & $<0,014$ & 52 & 1600 & 10,1 & 24,8 & 850 \\
\hline Hormiga Lodge & - & - & - & - & - & - & - & - & - & - \\
\hline Cuyabeno Lodge & 0,8 & 12 & 37 & 1,11 & $<0,014$ & 15 & 33 & 8,3 & 25,8 & 1510 \\
\hline \multirow[t]{2}{*}{$\begin{array}{l}\text { Referencia } \\
\text { TULSMA } \\
\end{array}$} & 30 & 100 & 200 & 10 & 0,5 & 1000 & 2000 & $6 a 9$ & $25 \pm 3$ & $50-750$ \\
\hline & \multicolumn{4}{|c|}{ Cumple la norma } & & \multicolumn{5}{|c|}{ No cumple la norma } \\
\hline
\end{tabular}

\section{Características Organolépticas (primer muestreo)}

En cuanto a las características organolépticas, todos los biodigestores estudiados, presentan un cambio de color en sus aguas analizadas, que van del amarillo, café al marrón. La mayoría de biodigestores analizados, no presentan malos olores, a excepción de Guacamayo Lodge en sus dos biodigestores, Siona Lodge en su biodigestor 1 y Hormiga Lodge en su biodigestor de hormigón, especialmente este último, donde los olores son bastantes fuertes.

La presencia de natas se identificó en los biodigestores 1 de Siona Lodge y Hormiga Lodge, como se observa en la Tabla 4.

Tabla 4. Características organolépticas

\begin{tabular}{lcccccc}
\hline \multirow{2}{*}{ Lodges } & \multicolumn{6}{c}{ Características Organolépticas } \\
\cline { 2 - 8 } & \multicolumn{2}{c}{ Color } & \multicolumn{2}{c}{ Olor } & \multicolumn{2}{c}{$\begin{array}{c}\text { Presencia de } \\
\text { Natas }\end{array}$} \\
\cline { 2 - 8 } Ecolodge Magic River & Amarillo & Amarillo & Ninguno & Ninguno & No & No \\
\hline Jamu Lodge & Café & Café & Mal olor & Ninguno & Sí & No \\
\hline Tapir Lodge & Amarillo & Amarillo & Ninguno & Ninguno & No & No \\
\hline Samona Lodge & Amarillo & Amarillo & Ninguno & Ninguno & No & No \\
\hline Guacamayo Lodge & & & Desagradable & Desagradable & No & No \\
\hline Siona Lodge & Crema & Café & $\begin{array}{c}\text { Fuerte } \\
\text { Desagradable }\end{array}$ & No & $\begin{array}{c}\text { Sí densa } \\
\text { capa }\end{array}$ & No \\
\hline Caimán Lodge & Café & Amarillo & No & No & No & No \\
\hline Hormiga Lodge & Café & - & $\begin{array}{c}\text { Fuerte } \\
\text { Desagradable }\end{array}$ & - & $\begin{array}{c}\text { Sí densa } \\
\text { capa }\end{array}$ & . \\
\hline Cuyabeno Lodge & Amarillo & \multirow{2}{*}{ Marrón } & No & No & No & No \\
\hline
\end{tabular}




\section{Segundo Muestreo}

En el segundo muestreo, los parámetros: nitratos y nitritos, sulfatos y temperatura son los únicos que cumplen la norma en todos los biodigestores analizados; en el resto de parámetros hay una variación del cumplimiento en los diferentes lodges, ver Tabla 5.

\section{Análisis de la capacidad de los biodigestores instalados}

Existen 29 biodigestores Rotoplas y tres de hormigón, instalados en los nueve lodges estudiados. De acuerdo con las recomendaciones del fabricante de los biodigestores Rotoplas, los biodigestores instalados (500-600 litros), tienen una capacidad para 5 usuarios cada uno (aportación en zona rural de 130 litros/día/usuario) (Rotoplas, 2015). Esto determina que la mayoría de lodges tienen una sobrecarga de usuarios, para el procesamiento normal de los biodigestores, como se demuestra en la tabla siguiente.

Tabla 5. Capacidad de biodigestores por lodge

\begin{tabular}{lcccccc}
\hline Descripción & $\begin{array}{c}\text { Número } \\
\text { Biodiges- } \\
\text { tores } \\
\text { Rotoplas }\end{array}$ & $\begin{array}{c}\text { Volumen } \\
\text { Biodigestor }\end{array}$ & $\begin{array}{c}\text { Capacidad } \\
\text { Usuarios } \\
\text { por } \\
\text { biodigestor }\end{array}$ & $\begin{array}{c}\text { Total } \\
\text { usuarios por } \\
\text { Lodges }\end{array}$ & $\begin{array}{c}\text { Capacidad } \\
\text { Biodigestores } \\
\text { por lodge }\end{array}$ & $\begin{array}{c}\text { Sobre } \\
\text { Carga } \\
\text { Usuarios }\end{array}$ \\
\hline $\begin{array}{l}\text { Ecolodge Magic } \\
\text { River }\end{array}$ & 2 & $500 \mathrm{~L}$ & 5 & 20 & 10 & 10 \\
\hline Jamu Lodge & 2 & $500 \mathrm{~L}$ & 5 & 56 & 10 & 46 \\
\hline Tapir Lodge & 5 & $500 \mathrm{~L}$ & 5 & 45 & 25 & 20 \\
\hline Samona Lodge & 4 & $500 \mathrm{~L}$ & 5 & 43 & 20 & 23 \\
\hline $\begin{array}{l}\text { Guacamayo } \\
\text { Ecolodge }\end{array}$ & 4 & $1000 \mathrm{~L}$ & 10 & 56 & 40 & 16 \\
\hline Siona Lodge & 4 & $600 \mathrm{~L}$ & 5 & 27 & 20 & 7 \\
\hline Caiman Lodge & 5 & $600 \mathrm{~L}$ & 5 & 33 & 25 & 8 \\
\hline Hormiga Lodge & - & - & - & - & - & - \\
\hline Cuyabeno Lodge & 3 & $600 \mathrm{~L}$ & 5 & 49 & 15 & 34 \\
\hline Totales & $\mathbf{2 9}$ & & & $\mathbf{3 2 9}$ & $\mathbf{1 6 5}$ & $\mathbf{1 6 4}$ \\
\hline Fut & & & &
\end{tabular}

Fuente: Rotoplas. Elaboración: Autores

${ }^{*}$ ) Incluye visitantes más personal administrativo 
Tabla 6. Resultados del segundo muestreo

Fuente: Resultados de análisis de laboratorio. Elaboración: Autores

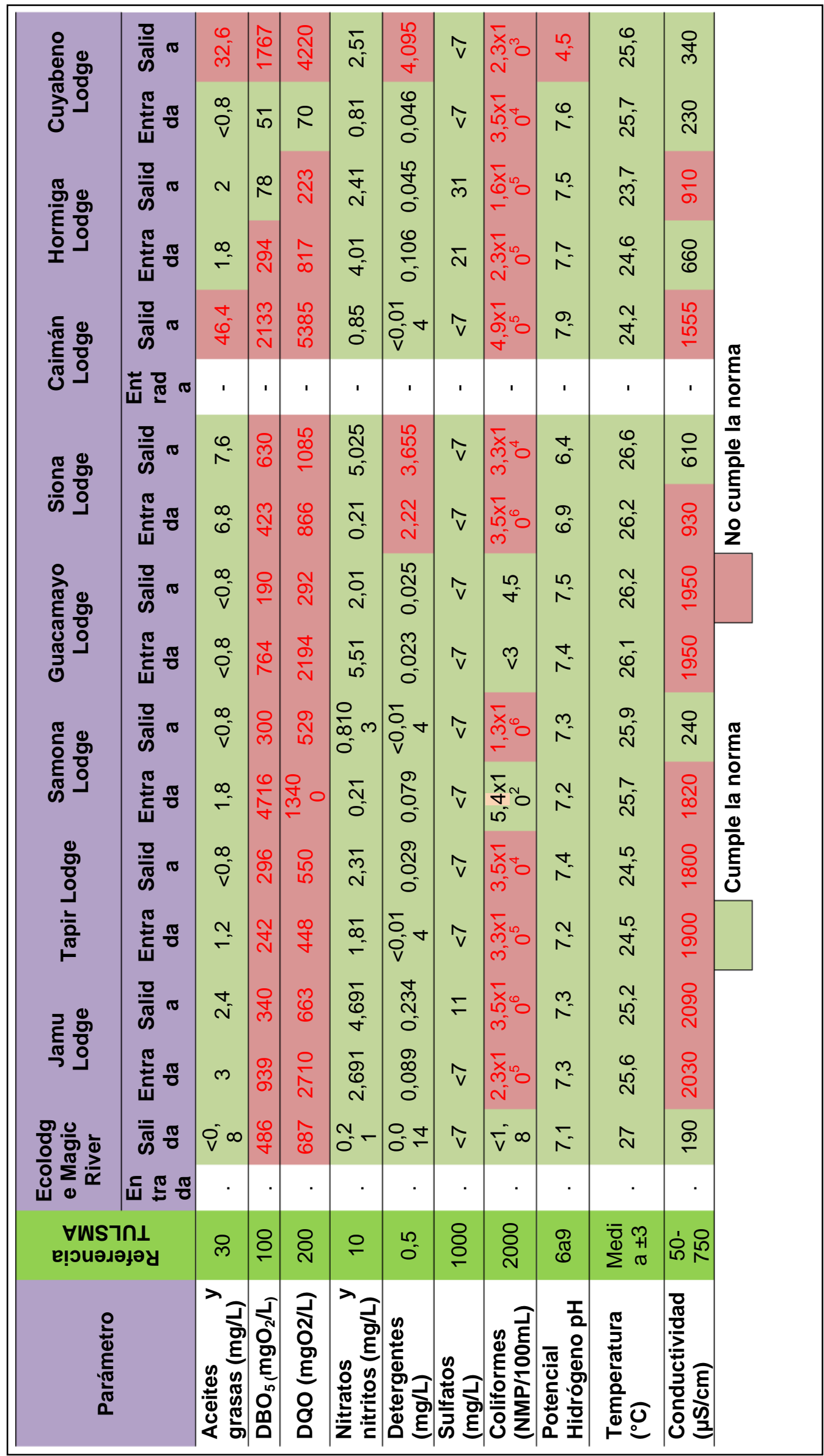




\section{Conclusiones y Recomendaciones}

a) Los biodigestores analizados en los lodges, incumplen con la normativa del TULSMA sobre "Límites de descarga a un cuerpo de agua dulce", al menos en uno de los parámetros examinados.

b) De acuerdo con las recomendaciones del fabricante de los biodigestores Rotoplas, la mayoría de lodges tienen una sobrecarga de usuarios, para el procesamiento normal de los biodigestores y no cumplen con las especificaciones respecto a su instalación.

c) El número de biodigestores debe estar en relación con la capacidad del lodge y el número de turistas con los cuales opera. Se debe analizar la capacidad de carga de los biodigestores instalados, a fin de verificar si corresponde con el número de turistas autorizados en cada lodge.

d) Los biodigestores no deben estar ubicados en zonas inundables y deben contar con adecuaciones complementarias para la descarga del agua tratada; que incluya un sistema de cloración y un pozo de adsorción o zanja de infiltración, antes de ser devuelta al ambiente.

e) Según los resultados de los análisis, se establece que los biodigestores están funcionando; sin embargo, en algunos casos el proceso no es suficiente para que los efluentes se ajusten a los valores que exige la norma vigente.

f) Se debe ampliar el estudio de los biodigestores en cada lodge, considerando todos los biodigestores instalados y nuevas variables: épocas de alta y baja pluviosidad, temporada alta y baja de visitas, número de turistas, características de los biodigestores, entre otras, que aporten al mejoramiento del sistema de biodigestores en la Reserva de Producción de Fauna Cuyabeno.

g) Cada lodge debe contar con un manual de mantenimiento de sus biodigestores y de capacitación periódica a los administradores y personal a cargo.

h) Elaborar un manual de buenas prácticas ambientales para la Reserva, que incluya normativas sobre el uso de jabones, detergentes y otros productos químicos que pueden afectar los recursos hídricos del Área Protegida y el funcionamiento de los biodigestores.

i) El buen uso y mantenimiento de los biodigestores deben ser parte de las normas y recomendaciones para protección de la Reserva y constar en la Patente de Operación Turística que emite el MAE.

j) La Administración de la Reserva debe realizar un programa de seguimiento permanente a los biodigestores instalados en los lodges, a través de formatos elaborados que incluyan: inspección visual del estado físico de biodigestores, tuberías y cajas de revisión, análisis de las descargas, verificación del mantenimiento, capacidad de carga de los biodigestores en relación con el número de turistas autorizado por el MAE, entre otros.

k) Realizar las adecuaciones necesarias para complementar el sistema de tratamiento de los biodigestores, cuyas aguas deben ser descargadas a un pozo de adsorción o zanja de 
infiltración; adicionalmente, se recomienda la instalación de un sistema de cloración, ubicado entre la salida del biodigestor y el pozo de adsorción.

I) Se deben realizar las adecuaciones necesarias, tanto para la "purga" del lodo acumulado y digerido por cada biodigestor; así como para el manejo de este material, hasta su disposición final en un relleno sanitario, conforme a las recomendaciones de los fabricantes de los biodigestores.

m) Reubicar los biodigestores que están en zonas inundables, elevando su nivel, para evitar se cubran con agua en la época de lluvia.

n) Revisar la existencia y funcionamiento adecuado de trampas de grasa para las aguas de cocina de todos los lodges; así como el buen estado de las cajas de revisión de los biodigestores.

\section{Bibliografía}

Andrade, C. V. (marzo de 2010). Propuesta de Creación de un Centro Turístico y de Interpretación Ambiental para la Reserva de Producción Faunística Cuyabeno en el Cantón Nueva Loja. Quito: UNIVERSIDAD TECNOLOGICA EQUINOCCIAL.

ECOLAP-MAE. (2007). Guía del Patrimonio de Áreas Naturales Protegidas del Ecuador. Quito, Ecuador: ECOLAP-USFQ, MAE, ECOFOUND (FAN), IGM. Primera Edición.

EPA. (1999). Standard Methods for the Examination of water and Wastewater. Obtenido de http//,mwa.co.th/download/file_upload/SMWW_1000-3000.pdf.

INEN. (2013). Norma Técinca Ecuatoriana Volunttaria NTE INEN 2169:2013 Agua. Calidad del Agua. Muestreo. Manejo y Conservación de Muestras. Quito: Instituto Nacional Ecuatoriano de Normalización. Obtenido de Instituto Ecuatoriano de Normalización. Quito, Ecuador

MAE. (2015). Anexos del Texto Unificado de Legislación Secundaria del Ministerio del Ambiente.

Quito: Registro Oficial Nro. 387. Edición Especial de 4 de noviembre de 2015.

Ministerio del Ambiente. (2012). Plan de Manejo Reserva de Producción de Fauna Cuyabeno. Quito, Ecuador. 
Robbins , J., \& Long, M. (noviembre de 2010). Reserva de Producción Faunística Cuyabeno. Recuperado el 10 de agosto de 2014, de Reserva de Producción Faunística Cuyabeno: http://cuyabeno.wordpress.com/fuentes/

Rotoplas. (2015). Manual Biodigestor. Sistema de Tratamiento de Aguas Residuales. Buenos Aires: ROTOPLAS ARGENTINA S.A. 